\title{
Animal experimental model of Peyronie's disease: A pilot study
}

\author{
Maria Angela Cerruto ${ }^{1}$, Carolina D'Elia ${ }^{1,2}$, Alberto Molinari ${ }^{1}$, \\ Francesca Maria Cavicchioli ${ }^{1}$, Antonio D'Amico ${ }^{1}$, Walter Artibani ${ }^{1}$ \\ ${ }^{1}$ Urology Clinic, A.O.U.I. Verona, Verona, Italy; \\ ${ }^{2}$ Urology Clinic, Santa Chiara Hospital, Trento, Italy.
}

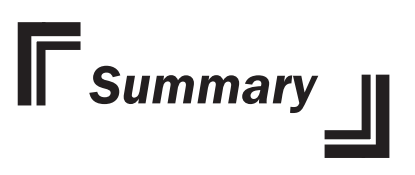

Objective: The Peyronie's disease (PD) is an idiopathic disorder of connective tissue of the penis, that involves the tunica albuginea of the corpora cavernosa and the adjacent areolar space. It is a growing clinical evidence to support the therapeutic potential of mesenchymal stem cells and histological findings has assumed a possible application of lipofilling technique in patients with PD. The objective of this experimental study is the creation of a murine experimental model of PD, evaluating with MRI the penis of the rats (feasibility study), in order to plane the application of lipofilling technique in an animal model.

Methods: Four male Wistar rats were anesthetized, fixed in prone position and subjected to MRI. The animals underwent, subsequently, an injection of thrombin in the tunica albuginea and MRI images were acquired at 7 and 21 days after injection with incision of the dartos.

Results: The MRI acquisitions, both in coronal and axial projection, showed an adequate visibility of the anatomical structures. At 7 days after thrombin injection with the dartos incision it was evident an oedematous portion, visible as a hyperintense area, located at the injection area. At 21 days after injection, oedema was partially resolved: the injection part of the hyperintense area remains unchanged, while the remaining area appears to be part of a re-absorption and re-organization process.

Conclusions: Since none of the various treatment modalities currently available for the management of PD is able to bring healing, the researchers' attention is increasingly directed towards innovative treatment programs, such as the use of stem cells of mesenchymal origin. At the present time, the research in PD is hampered by the lack of universally accepted animal model and this is likely attributed to the limited insight into PD mechanisms and the difficulties faced by current animal models to truly represent the complexity.

KEY WORDS: Peyronie's disease; Murine animal model.

\section{INTRODUCTION}

The Peyronie's disease (PD) is an idiopathic disorder of connective tissue of the penis, that involves the tunica albuginea of the corpora cavernosa and the adjacent areolar space. A fibrous plaque replaces the elastic fibers that normally constitute the tunica albuginea, limiting its extensibility, and causing pain during erection, erectile dysfunction and difficulty of penetration, as a result of the deviation of the penis.

Although it was once considered a relatively infrequent disease, some studies show that the prevalence of IPP is comparable to that of diabetes or of urolithiasis $(1,2)$. A recent epidemiological study reported a prevalence of $3.2 \%$ (3), highlighting the potential psychosocial impact of this disease.

The Peyronie's disease affects predominantly adulthood (approximately $60 \%$ of cases between 40 and 60 years) and is still present at young age (10-20\% at the age of 40$)$ and advanced age (15-20\% over 60 years of age) (4). Further studies have tried to identify risk factors associated with PD: smoking, hypertension, hyperlipidemia 
have all been taken into account, but basically considered to be associated with erectile dysfunction more than strictly to PD.

Microscopically, the tunica albuginea is largely composed by fibrillar collagen type I, woven with elastin fibers, in order to form organized structures (5). Even the plaques of PD are mostly constituted from collagen type I and III (6), but the elastin component, which gives to the tunica albuginea elongation properties up to $150 \%$, is altered: the decrease elastin, as well the alterations in the arrangement of the tunic layers, are characteristics of PD, that can lead to deviations in penile erection and erectile dysfunction (7).

More recent papers have identified a strong association of PD with both Dupuytren's disease and the HLA B-27 (8). Other studies have taken into account immunological factors, showing that patients with PD in $75.8 \%$ of cases presented an abnormal immunological test, $48.5 \%$ had alterations in cell-mediated immunity and the $37.9 \%$ presented increase in markers for autoimmune diseases (9) but it seems that this predisposition, rather than systemic, is localized to the tunica albuginea. Another study has documented evidence of high anti-elastin antibodies in the serum of patients with IPP, hypothesizing, the same way, an autoimmune etiology (10).

The role of free radicals in the pathogenesis of plaque seems to be important; Bivalacqua et al. (11) have shown, on the basis of other studies (12-14), that the production of Nitric Oxyd (NO) can inhibit the deposition of collagen in the murine model of PD.

The most widely accepted theory considers the penile trauma as main causative factor in the pathogenesis of PD: an acute traumatic event or a repetitive microtrauma (e.g. during sexual activity) can result in delamination of the tunica albuginea and microvascular damage $(8,15)$. Fibrin was found to be potentially involved in the development of the fibrotic plaque in a murine model of PD (16). In the same study, and in another more recent (17), was also reported the presence of increased levels of PAI-1, an important inhibitor of fibrinolysis and collagenolysis: its increased activation appears to play a key role in the development of penile plaque.

The PD has been associated with many other conditions in urologic procedures such as radical retropubic prostatectomy, cystoscopy and introduction of indwelling urethral catheters, genital or peritoneal trauma, urethritis, lipomas (18).

Even atherosclerosis has been mentioned as a target area on the study of PD, due to the similarities about the pathophysiology (19), regarding the common mechanism of cell hyperproliferation, that leads to the formation of a plaque.

Since none of the various treatment modalities currently available for the management of PD is able to bring healing to the patients who are affected, the researchers' attention is increasingly directed towards innovative treatment programs based on a better understanding of the pathophysiological disease.

Moreover, the overexpression of cytokines and autoimmune and genetic factors have been cited as cofactors (8). The resulting inflammatory process would be responsible for the onset of a vicious circle of vascular injury, ischaemia, hyperpermeability, fibrosis and increased ischemia itself. This vicious circle can be broken using the proangiogenetic capacity of mesenchymal stem cells. It is a growing clinical evidence to support the therapeutic potential of mesenchymal stem cells for the revascularization of ischemic tissues and the recovery of their function.

In a recent italian study Rigotti et al. (20) have tested the effectiveness of purified lipoaspirates on 20 patients operated on for breast cancer with severe side effects and irreversible functional damage from postoperative radiotherapy.

60 to $80 \mathrm{cc}$ of adipose tissue were collected from the medial region of the knee, from the abdominal region or trochanteric: subsequently, the lipoaspirates was centrifuged at $2700 \mathrm{rpm}$ for 15 minutes, in order to remove most of the deposits of triglycerides and to cause injury of membrane in adipocytes, in order to allow the rapid clearance once injected. In this way, the study authors have created a sort of concentration of adult stem cells, which, maintained their three-dimensional microenvironment, appear to be more effective in the neoangiogenetic process (21).

In the same paper the ultrastructure of the tissue damaged by radiotherapy has been studied before injection of lipoaspirates and after 1, 2, 6 and 12 months.

Prior to injection, the tissue showed fibrotic patterns similar to those of scleroderma: duplication in the basement membrane of the capillaries, spaces between the endothelial cells, adipocytes containing lysosomes or clusters of mitochondria, accumulation of collagen.

The after-treatment ultrastructural analysis showed signs of neoangiogenetic, neoadipogenetic and antifibrotic activity, due to the activity of adult stem cells contained in lipoaspirates (22-24).

At a clinical level, this activity corresponds to a dramatic improvement of symptoms, disappearance of necrosis and wound healing.

Based on these innovative clinical and histological findings has assumed a possible application of lipofilling technique in patients with $\mathrm{PD}$.

It was therefore drawn up an experimental protocol consists of two basic steps:

1. creating an animal model of PD on the basis of experiments already conducted $(16,25)$ verifying with MRI (Magnetic Resonance Imaging) stages of development of the disease;

2. the application of lipofilling technique in an animal model.

The objective of this experimental study is the creation of a murine experimental model of IPP (16), evaluated with MRI (feasibility study).

\section{Materials AND MEthods}

Four male Wistar rats weighting between 420 and $450 \mathrm{~g}$. were fed with standard rodent diet (Rieper SpA, Italy) and maintained under conditions of controlled temperature and light, respecting the principles of the "NIH Guide for the Care and Use of Laboratory Animals" and the directives of the European Community. 
Before being subjected to MRI, the rats were anesthetized in an induction chamber by inhalation of a mixture $70 / 30 \%$ of nitrous oxide and oxygen containing 5\% isoflurane. During the execution of the examination, anesthesia was maintained via buccal mask with the same mixture of nitrous oxide and oxygen with isoflurane 3\%. Image acquisition was performed using a scanner Biospec (Bruker, Karlsruhe, Germany) equipped with a horizontal magnet operating at 4.7 Tesla with opening of $33 \mathrm{~cm}$ (Oxford Ltd, Oxford, UK) (Figure 1). The rats were fixed in a prone position on a birdcage type coil diameter of $7.2 \mathrm{~cm}$ (Figure 2).

In order to verify the correct positioning of the animal with respect to the coil, Tl-weighted coronal projection acquisitions were performed, while, in order to obtain pre-and post-injection anatomical details, acquisitions T2-weighted images in axial view were performed.

The coronal images were acquired with a relaxation time (TR) of $486 \mathrm{~ms}$, echo time (TE) of $11 \mathrm{~ms}$, with the use of 2 medium for 20 slices, each of thickness $1 \mathrm{~mm}$. The field of view (FOV) was $6 \times 6 \mathrm{~cm}$ and the matrix choice of $256 \times 256$, corresponding to a spatial resolution of $0234 \mathrm{~mm}^{2} /$ pixel.

Axial images were acquired with a relaxation time (TR) of $5000 \mathrm{~ms}$, echo time (TE) of $67 \mathrm{~ms}$, with the use of 2 medium for 25 slices, each of thickness $1 \mathrm{~mm}$. The field of view (FOV) was $6 \mathrm{~cm} \times 3$ matrix and the choice of $512 \times 256$, corresponding to a spatial resolution of $0.117 \mathrm{~mm}^{2} /$ pixel.

The animals underwent, subsequently, an injection of 60 $\mathrm{mL}$ of thrombin in the tunica albuginea, using a $1 \mathrm{~mL}$ syringe with $30 \mathrm{G}$ needle. In the first measure, the thrombin was injected without opening the dartos (Figure 3): thereafter, considering the substantial ineffectiveness of the first surgery, an incision was performed along the dartos of about $2 \mathrm{~mm}$ along the penile axis, in order to have a more secure access to the subdartoic spaces, and the thrombin was injected with the same method (Figure 4). MRI images were acquired in the same manner as described above at 7 and 21 days after injection with incision of the dartos.

Figure 1.

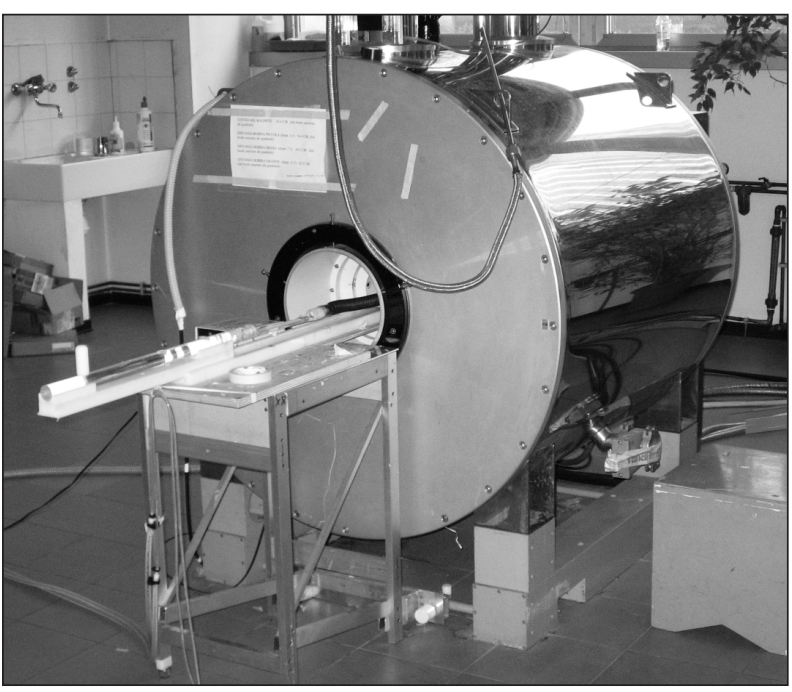

Figure 2.

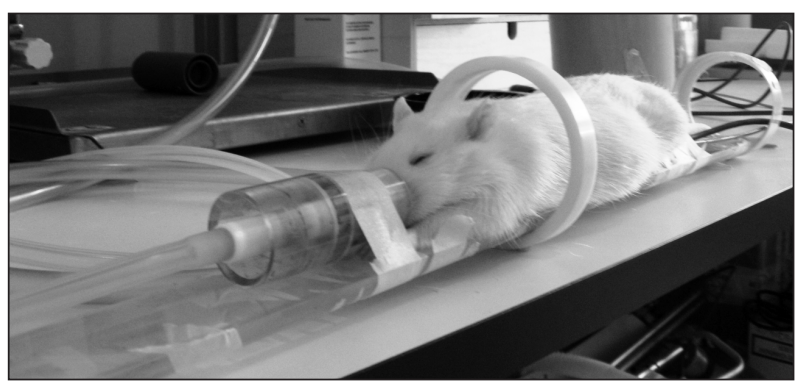

Figure 3.

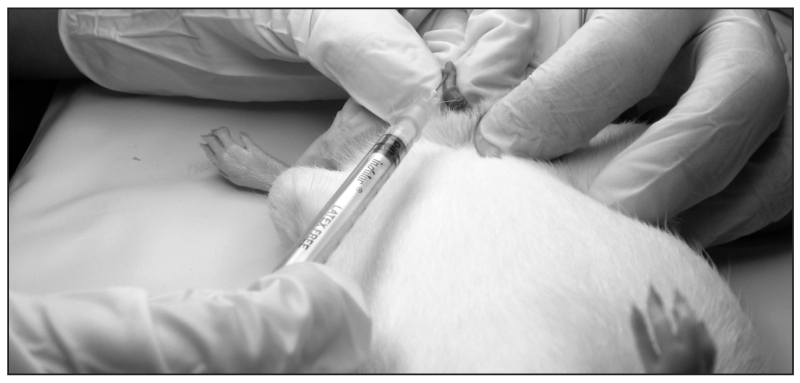

Figure 4.

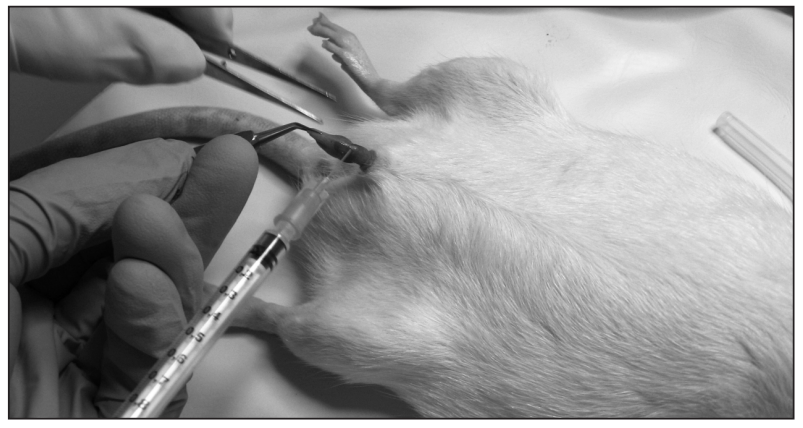

\section{Results}

The acquisitions, both in projection coronal both in axial, have demonstrated adequate visibility of anatomical structures.

Figure 5 shows a T1-weighted image, a coronal projection of the penis, while Figure 6, T2-weighted axial view, shows the results produced by the thrombin injection, offering a good view of the albuginea and its relationship with the superficial and deeper structures.

At 7 days after thrombin injection with incision of the dartos it was evident a edematous portion (Figure 7) located at the injection area. Oedema, visible as a hyperintense area, appeared to affect the section previously seen as a hypointense rim surrounding the corpora cavernosa of the penis, leaving them intact and with a signal intensity comparable to that seen in the image acquired before the injection.

At 21 days after injection, edema was partially resolved (Figure 8): in the injection part of the hyperintense area remains unchanged, while the remaining area appears to be part of a reabsorption and reorganization process. 
Figure 5.

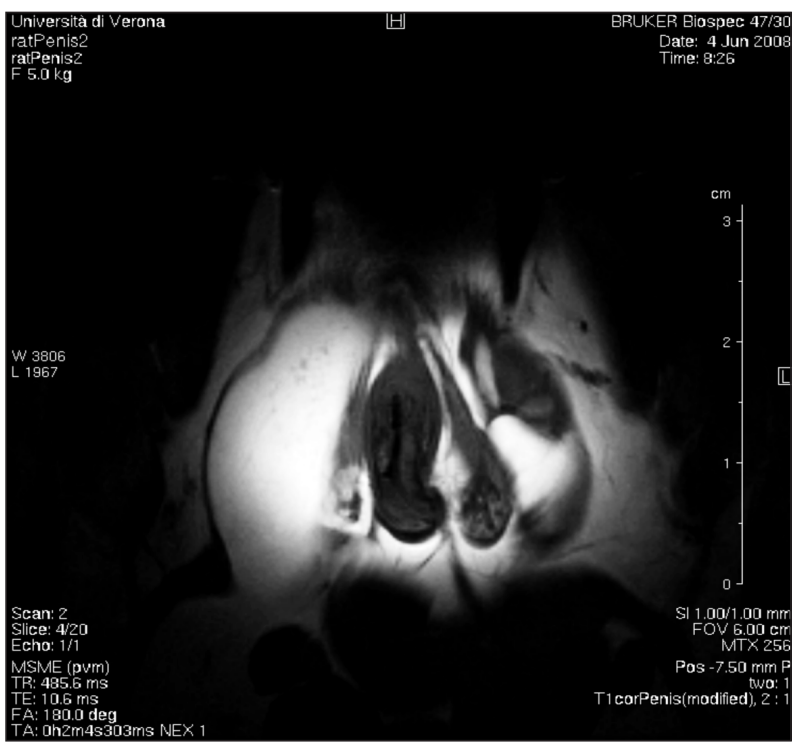

Figure 7.

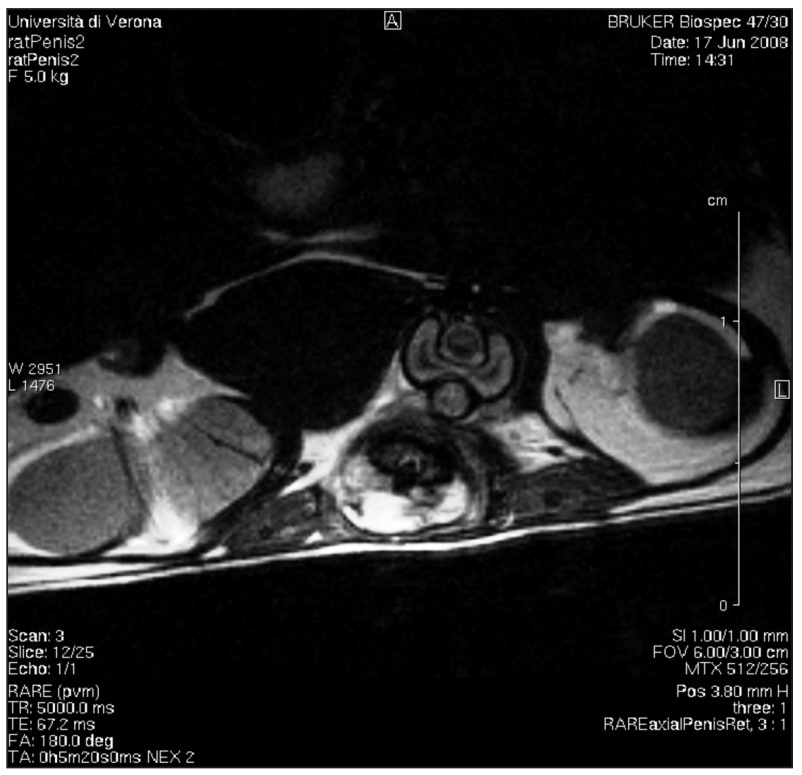

\section{Discussion}

The low success rate obtained by standard therapies has fueled our interest in the development of new treatment options, such as the use of stem cells of mesenchymal origin. In literature has recently emerged a growing increase of the clinical evidence to support the therapeutic potential of mesenchymal stem cells for the revascularization of ischemic tissues and the recovery of their function (20).

Significant clinical results were obtained from autologous endothelial and hematopoietic stem cells from the bone marrow in ischemic lesions at the level of the limbs, myocardium and of the retina in vitro and in animal models, thereby elucidating the mechanisms of stem cell - mediated neo-angiogenesis.

The use of stem cells could be useful for the management
Figure 6.

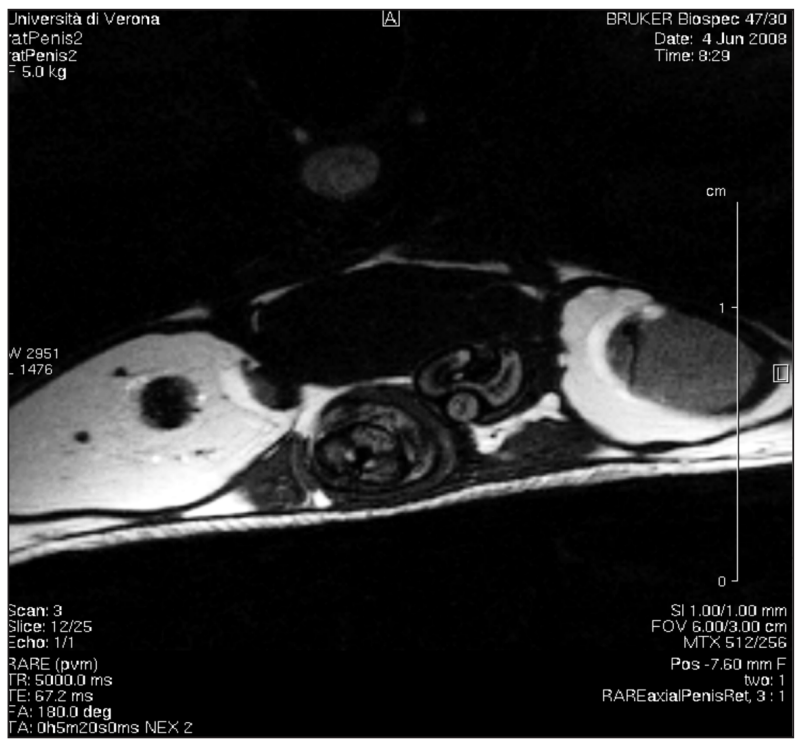

Figure 8.

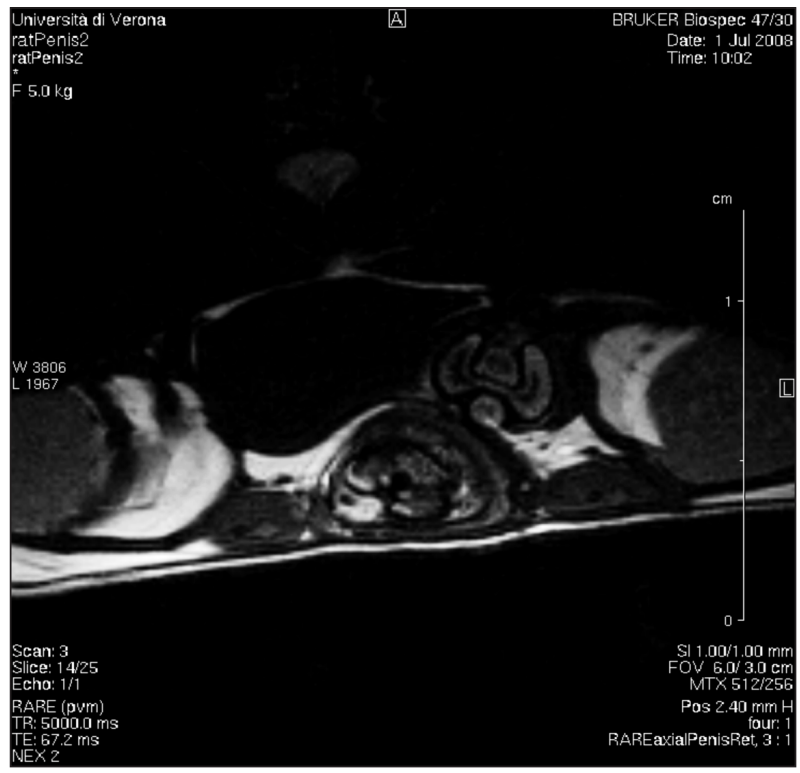

of fibrotic connective tissue diseases such as PD, breaking a vicious circle of self-perpetuating inflammatory condition with post-traumatic vascular injury, subsequent ischemia, hyperpermeability, increased fibrosis and ischemia itself. Since there is no data in the literature concerning the role of stem cells in management of $\mathrm{PD}$, we planned to develop a murine model of stable IPP, monitored with imaging (MRI), and then try to corroborate the hypothesis that mesenchymal stem cells can have a positive influence on the natural history of this disease.

In order to create a murin model of PD we injected $60 \mu \mathrm{l}$ of thrombin at the level of the tunica albuginea obtaining after 3 weeks after injection the persistence of the inflammatory reaction at the site of inoculation, identifi- 
able to control MRI, without secure evidence of fibrosis. Moreover, a review of the literature on human MRI of the penis was carried out, in order to assess the feasibility of the application of this method in animals: although ultrasound is the primary mode of imaging penis, the MRI may use a higher spatial resolution and improved contrast soft tissue. MRI is to be considered as an opportunity for progress in penile imaging and is able to solve clinical questions for which the ultrasound is not conclusive.

In addition to PD, other diseases can be investigated by this method, such as primitive or secondary penile neoplasms, periurethral abscess, venous thrombosis, fractures; can also be rated the positioning of penile prostheses after surgery.

The review of the literature has concerned in particular the possibility to discriminate with the MRI the tunica albuginea compared to the remaining structures in humans, so as to apply sequences of acquisition and anatomical similarities also in rats.

The T2-weighted images provide more detailed anatomical details: in particular, the tunica albuginea is visible as a hypointense area that surrounds the corpora cavernosa and which also includes Buck's fascia.

In a work of Pretorius et al. (26) also the PD was studied by magnetic resonance imaging: there was evidence of a thickening of the tunica albuginea, visible in both T2weighted both in those T1- weighted images.

Davila et al. (16) have recently proposed an animal model of IPP injecting at the level of the tunica albuginea of rat $30 \mu \mathrm{l}$ of fibrin and thrombin, inducing edema after one week after inoculation and fibrosis after three weeks. The use of these components would trigger the final steps of the coagulation cascade and reproduces experimentally what happens after a trauma at the level of the tunica albuginea. The profibrotic effect is most likely due to the fibrin and thrombin deposition in a fibroblasts rich and hypovascularized tissue.

The study of Davila et al. also showed that, compared to previous methods that used injection of TGF- $\beta 1$ (27), the fibrin induced plaque develops more quickly (three weeks instead of six weeks). The TGF- $\beta 1$, in fact, has an anti-inflammatory action in reducing macrophage activation and inhibition of cytotoxic T cells $(28,29)$ : this may explain the study of El-Sakka et al. (27) the non-formation of plaque in three weeks and the discovery of the same six weeks, the latter a result of the action of TGF$\beta 1$ profibrotic same.

In our study, after this period there has been found the persistence of the inflammatory condition (edema), probably due to the lower speed of action of thrombin compared to fibrin alone. It is important to observe, that the literature data are only based on histological founds, not also on imaging, as in our study.

While the exact mechanism of Peyronie's disease (PD) remains an enigma, the pathophysiology of $\mathrm{PD}$ is considered to be multifactorial, with interactions of genetic predisposition, trauma, tissue inflammation and aberrant wound healing.

The most frequently reported models of PD can be classified as TGF $\beta 1$, fibrin and surgical trauma-induced models. In vitro studies using Peyronie's fibroblast culture media have also provided further insights into cel- lular mechanism of PD. At the present time, the research in PD is hampered by the lack of universally accepted animal model and this is likely attributed to the limited insight into PD mechanisms and the difficulties faced by current animal models to truly represent the complexity and complete spectrum of human disease.

\section{REFERENCES}

1. Smith CJ, McMahon C, Shabsigh R. Peyronie' disease: the epidemiology, aetiology and clinical evaluation of deformity. BJU Int. 2005; 95:729-732.

2. Sommer F, Schwarzer U, Wassmer G. et al. Epidemiology of Peyronie's disease. Int J Impot Res. 2002; 14:379-383.

3. Schwarzer U, Sommer F, Klotz T, et al. The prevalence of Peyronie's disease: results of a large survey. BJU Int. 2001; 88:727-730.

4. Pryor JP, Ralph DJ. Clinical presentation of Peyronie's disease. Int J Impot Res. 2002; 14:414-417.

5. Gentile V, Modesti A, La Pera G, et al. Ultrastructural and immunoistochemical characterization of the tunica albuginea in Peyronie' disease and veno-occlusive function. J Androl. 1996; 17:96-103.

6. Somers KD, Sismour EN, Wright GL Jr, et al. Isolation and characterization of collagen in Peyronie's disease. J Urol. 1989; 141:629-631.

7. Akkus E, Carrier S, Baba K, et al. Structural alterations in the tunica albuginea of the penis: impact of Peyronie's disease, ageing and impotence. Br J Urol. 1997; 79:47-53.

8. Mulhall JP. Expanding the paradigm for plaque development in Peyronie's disease. Int J Impot Res. 2003; 15 (Suppl. 5): S93-102.

9. Schiavino D, Sasso F, Nucera E, et al. Immunologic findings in Peyronie's disease: a controlled study. Urology. 1997; 50:764-768.

10. Stewart S, Malto M, Sandberg L, Colburn KK. Increased serum levels of anti-elastin antibodies in patients with Peyronie's disease. J Urol. 1994; 152:105-106.

11. Bivalacqua TJ, Champion HC, Hellstrom WJG. Implications of nitric oxide synthase isoforms in the pathophisiology of Peyronie's disease. Int J Impot Res. 2002; 14:345-352.

12. Ferrini $M$, et al. Inducible nitric oxide synthase (iNOS) and hemoxygenase (HO-1) in the Peyronie's plaque. J Urol. 2001; 154(suppl.):832A.

13. Bivalacqua TJ, Champion HC, Leungwattanakij S, et al. Evaluation of nitric oxide synthase and arginase in the induction of Peyronie's-like condition in the rat. J Androl. 2001; 22:497-506.

14. Khan MA, Thompson CS, Mumtaz FH, et al. The effect of nitric oxide and peroxynitrite on rabbit cavernosal smooth muscle relaxation. World J Urol. 2001; 19:220-224.

15. Devine CJ Jr, Somers KD, Jordan SG, Sclossberg SM. Proposal: trauma as the cause of the Peyronie's lesion. J Urol. 1997; 157:285290

16. Davila HH, Ferrini MG, Rajfer J, Gonzalez-Cadavid NF. Fibrin as an inducer of fibrosis in the tunica albuginea of the rat: a new animal model of Peyronie's disease. BJU Int. 2003; 91:830-838.

17. Davila HH, Magee TR, Zuniga FI, et al. Peyronie's disease associated with increase in plasminogen activator inhibitor in fibrotic plaque. Urology. 2005; 65:645-648.

18. Gholami SS, Gonzalez-Cadavid NF, Lin CS, et al. Peyronie's disease: a review. J Urol. 2003; 169:1234-1241.

19. Mulhall JP, Anderson MS, Lubrano T, Shankey TV. Peyronie's 
disease cell culture models: phenotypic, genotypic and functional analyses. Int J Impot Res. 2002; 14: 397-405.

20. Rigotti G, Marchi A, Galiè M, et al. Clinical treatment of radiotherapy tissue damage by lipoaspirate transplant: a healing process mediated by adipose-derived adult stem cells. Plast Reconstr Surg. 2007; 119:1409-1422; discussion 1423-1424.

21. Scherberich A, Beretz A. Culture of vascular cells in tridimensional (3-D) collagen: a methodological review. Therapie. 2000; 55:35.

22. Rehman J, Traktuev D, Li J, et al. Secretion of angiogenic and antiapoptotic factors by human adipose stromal cells. Circulation. 2004; 109:1292.

23. Rafii S, Lyden D. Therapeutic stem and progenitor cell transplantation for organ vascularization and regeneration. Nat Med. 2003; 9:702.

24. Peroni D, Scambi I, Pasini A, et al. Stem molecular signature of adipose-derived stromal cells. Exp Cell Res. 2007; 10:1-13.
25. Ferrini MG, Kovanecz I, Nolazco G, et al. Effects of long-term vardenafil treatment on the development of fibrotic plaques in a rat model of Peyronie's disease. BJU Int 2006; 97:625-633.

26. Pretorius ES, Siegelman ES, Ramchandani P, Banner MP. MR imaging of the penis. RadioGraphics. 2001; 21:S283-299.

27. El-Sakka AI, Hassoba HM, Chui RM, et al. An animal model for Peyronie's-like condition associated with an increase of transforming growth factor beta mRNA and protein expression. J Urol. 1997; 158:2284-2290.

28. Tsunawaki S, Sporn M, Ding A, Nathan C. Deactivation of macrophages by transforming growth factor-beta. Nature. 1988; 334:260-262.

29. Ranges GE, Figari IS, Espevik T, Palladino MA Jr. Inhibition of cytotoxic $T$ cell development by transforming growth factor beta and reversal by recombinant tumor necrosis factor alpha. J Exp Med. 1987; 166:991-998.

\section{Correspondence}

Maria Angela Cerruto, MD, FEBU

mariaangela.cerruto@univr.it

Carolina D'Elia, MD, FEBU (Corresponding Author)

karolinedelia@gmail.com

Alberto Molinari, MD

yefet83@yahoo.it

Francesca Maria Cavicchioli, MD

francesca.cavicchioli@gmail.com

Antonio D'Amico, MD

antonio.damico@ospedaleuniverona.it

Prof. Walter Artibani, MD

walter.artibani@univr.it

Urology Clinic, A.O.U.I. Verona

P.zza L.A. Scuro 10 - 37134 Verona, Italy 